\title{
Marco teórico para una proyección marítima desde una perspectiva oceánica integrada
}

\author{
MILAN MARINOVIC PINO
}

$>$ Doctor en Sociología y Ciencias Políticas, Universidad Pontificia de Salamanca, España. Universidad de Valparaíso, Chile

milan.marinovic@uv.cl

ORCID 0000-0002-2275-0027

Universidad de Valparaíso

Facultad de Arquitectura

Revista Márgenes

Espacio Arte Sociedad

Marco teórico para una proyección marítima desde una perspectiva

oceánica integrada

Octubre 2021 Vol. $14 \mathrm{~N}^{\circ} 20$

Páginas 7 a 14

ISSN elec. 0719-4463

Recepción: Diciembre 2020

Aceptación: Abril 2021

doi.org/10.22370/marge-

nes.2021.14.20.2994

\section{RESUMEN}

El dilema existencial que conlleva el fenómeno de la globalización y los efectos del impacto tecnológico, se hace explícito en la necesidad de una mirada sistémica para abordar la realidad marítima y la necesidad de asumir la interacción marítima con una conciencia moral asociado al desarrollo marítimo -objetivo de este trabajo- con una base existencial para una solución rápida al deterioro progresivo que se observa en las condiciones primarias de estabilidad social del mundo emergente.

La visión sistémica de la interacción marítima y los conceptos de eco-océano, océano-política y mar presencial asumidos en términos relacionales y fundamentados en los principios vitorianos de la tesis sobre la paz dinámica, en cuanto al resto del papel de las Fuerzas Armadas para garantizar la paz y las hipótesis de conflicto que surgen de las amenazas percibidas, sugieren la incorporación de una nueva teoría en términos de desarrollo.

\section{PALABRAS CLAVE}

desarrollo marítimo, globalización, visión sistémica, eco-océano, Francisco de Vitoria

\section{Theoretical framework for a maritime projection from an integra- ted ocean perspective}

\section{ABSTRACT}

The existencial dilemma that carries the phenomenon of globalization and the effects of technological impact, becomes explicit in the necessity of a sistemic view to approach maritime reality and the need to assume maritime interaction with a moral conscience associated to maritime development -the aim of this paper - with an existential base for a quick solution to progressive deterioration that can be observed in the primary conditions of social stability of the emerging world.

The sistemic view of maritime interaction and the concepts of eco-ocean, ocean-politics, and presential sea assumed in relational terms and founded on the Vitorian principles of the thesis on dynamic peace, as far as the remainder of the role of the Armed Forces to ensure peace and the hypotheses of conflict that come about from perceived threats are concerned, suggest the incorporation of a new theory in terms of development.

\section{KEYWORDS}

maritime development, globalization, systemic vision, eco-ocean, Francisco de Vitoria 


\section{INTRODUCCIÓN}

La naturaleza humana y social del hombre frente al desafío de acceder a la plenitud de su realidad marítima, y ante el imperativo nacional de desplegar sus capacidades para el desarrollo del potencial natural de sus recursos plantean a la sociedad y los individuos que la conforman, la premisa existencial de que ello no será posible si no se es parte de un esfuerzo humano colectivo.

Todo colectivo humano como cuerpo social estructurado en función de objetivos o metas de desarrollo, no tiene realidad si en la satisfacción de sus necesidades primarias, de naturaleza individual y colectiva, no cuenta con una base de estabilidad social que lo proyecte y con acciones colectivas que lo materialicen en el marco de una filosofía existencial, en función de sus posibilidades en equilibrio con las variables de su entorno.

La estabilidad social de los sistemas, como clima organizacional no se materializa ni perdura en el tiempo si como entorno psicosocial, no cuenta con el compromiso de acciones colectivas que los aglutine y que estructuradas en función de sus necesidades primarias le otorgue al cuerpo social las bases de sustentación para su preservación, integración y desarrollo.

El ser colectivo como tal no existe, son las conductas individuales las que a través de iniciativas y mecanismos formales que la encausan las que actúan y que concatenadas en la forma de conciencia orgánica se hacen presentes en un sistema de creencias y de valores que explícitos en su dimensión espacial y temporal dan pauta de acción a las necesidades prácticas en la línea de su desarrollo histórico.

Como consideración sociológica, entonces, para la elaboración de un marco teórico en el desarrollo de una política marítima desde una perspectiva oceánica integral, observamos en consecuencia la necesidad de visualizar la actividad marítima como sistema en el contexto de un todo relacional sustentada en los parámetros culturales estructurados que dan vida existencial al quehacer marítimo, y de identificar las variables del entorno que en su dinámica de cambio conforman una realidad emergente, así como los fundamentos éticos que la contienen en un entorno de estabilidad social y marítima.

\section{PARÁMETROS EXISTENCIALES Y SU REALIDAD EMERGENTE}

El impacto del desarrollo tecnológico, manifestado en la última década por una creciente interconexión entre el mundo social y el natural, y la escala global que en el presente toman los fenómenos sociales, conllevan un flujo creciente de información que entregan a la humanidad la visión de un mundo cada vez más interdependiente y necesitado, y plantean un cambio de referencia existencial que sitúa al ser humano en actitud distinta a la asumida en su pasado.

Esta visión, que surge en el horizonte del tercer milenio, como resultado de la dinámica de cambios y toma de conciencia del impacto que hoy día tienen los fenómenos sociales, conlleva para la humanidad un nuevo marco de referencia existencial definido por el discurrir científico como "fenómeno de la globalización", y que, en lo político, lo social y lo económico, hace percibir su realidad exis tencial desde una perspectiva integral y en términos relacionales.

La visión de un mundo globalizado en los albores del siglo XXI, entrega a la humanidad la percepción de un distanciamiento creciente entre un mundo desarrollado y otro dependiente, y una brecha relacional entre la realidad social y el medio natural que lo contiene, con el progresivo impacto de su deterioro.

El dilema existencial que conlleva el fenómeno de la globalización y los efectos del impacto tecnológico, se hace explícito en la necesidad de una conciencia moral asociada a su desarrollo, que dé base existencial para una urgente solución al progresivo deterioro que se observa en las condiciones primarias de la estabilidad social del mundo emergente.

Como filosofía existencial, el sentido de este llamado pasa por la toma de conciencia individual y responsabilidad colectiva en la necesidad de conciliar la existencia de dos mundos: el dado por la naturaleza y el social edificado por el hombre, y en el imperativo de replantear su coexistencia en un mundo relacional en el que no solo importa las relaciones del hombre con su entorno asumido como lugar de vida o ámbito vital, sino también las relaciones del hombre con el hombre, asumido como comunidad de vida en un mundo social cada vez más necesitado e interdependiente.

Situar el problema de la globalización en torno a la tecnología, ética y valores, supone tomar conciencia acerca de los factores estáticos y dinámicos que conjugan la realidad social en la idea de lugar de vida y comunidad de vida, y de su interrelación en la interfase de los dos mundos que la contienen: el del mundo natural y el social, supone de una conciencia individual y responsabilidad colectiva que como filosofía existencial y precipitado cultural del desarrollo humano en el proceso de interacción social sustente la idea de calidad de vida.

Lugar de Vida, Comunidad de Vida y Calidad de Vida, percibido como un todo relacional en un contexto vivencial de conciencia individual y responsabilidad colectiva y en el que el grado de dependencia que surja de sus relaciones den cabida integral al mundo natural y al social, en un entorno de estabilidad son, a nuestro juicio, los parámetros existenciales que, desde la perspectiva vitoriana, otorgan a la realidad marítima emergente un marco de convivencia pacífica y que de cara al tercer milenio, plantean a la humanidad el desafío de su desarrollo.

\section{VISIÓN SISTÉMICA DE LA REALIDAD MARÍTIMA Y EL ECOCÉANO DEL PACÍFICO}

La Visión Sistémica de lo marítimo en el Pacífico plantea la exigencia de interpretar la realidad marítima de los países ribereños a su cuenca, como un todo armónico, en la que considerando sus aspectos estáticos y dinámicos siempre presentes en una realidad social, se integren la idea de lugar de vida y comunidad de vida.

Asimismo, la visión sistémica de su realidad marítima plantea la exigencia de asumirla en términos relacionales como un entramado socio-cultural de intereses y valores regulados por políticas de desarrollo nacionales, en las que las relaciones de poder son sus resultantes.

Por ecocéano del Pacífico, entendemos en consecuencia, el entorno de estabilidad social que surge de las interacciones marítimas de los Estados participantes en la Cuenca, y que en equilibrio con su entorno, integran los aspectos estáticos y dinámicos que caracterizan su realidad social y son comunes al área oceánica y marítima.

Desde la perspectiva de la Oceanopolítica, estas variables tienen su impacto en la calidad de vida marítima de las naciones ribereñas a la Cuenca. 


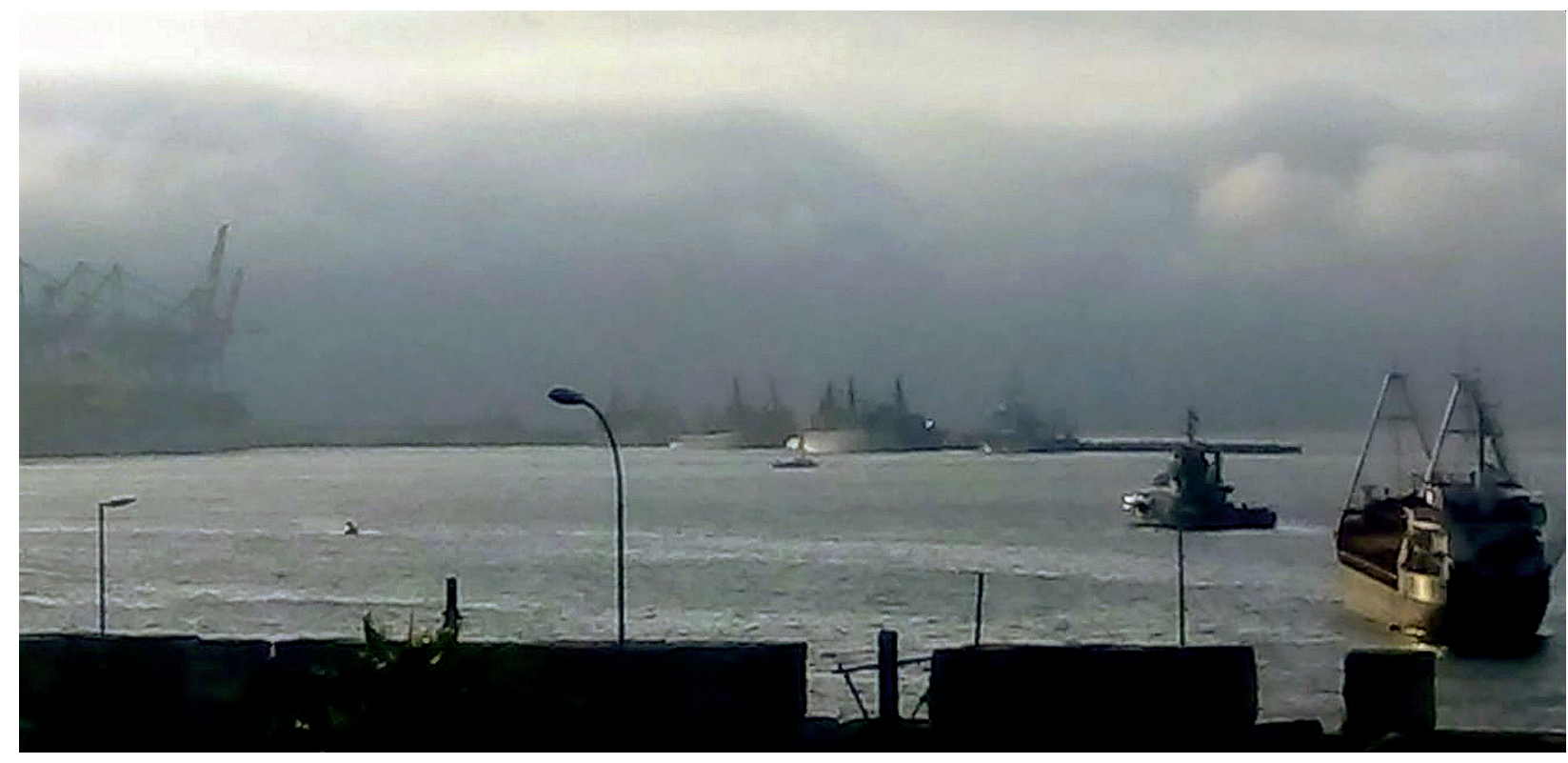

La Oceanopolítica del Pacífico como aproximación interdisciplinaria que incorpora el mar en la estructura del espacio político, desde una perspectiva relacional y visión sistémica, ofrece una respuesta válida del hemisferio austral para asumir el desafío del desarrollo marítimo.

La Oceanopolítica percibe la Cuenca del Pacífico como el mayor espacio marítimo del planeta donde convergen el $60 \%$ de la población mundial y donde se generan los $3 / 5$ del comercio global mundial y en cuya cuenca en el horizonte del Siglo XXI se configura el centro oceanopolítico de desarrollo mundial.

En este contexto, la dinámica de la interacción social concebida como entramado de intereses individuales y colectivos, presuponen pautas de conductas y situaciones potenciales de conflicto que, enraizadas en un sistema de creencias y valores, conllevan procesos de crisis y de tensión social en un entorno de inestabilidad social.

El ecocéano del Pacífico, en su visión sistémica y percibido en términos relacionales constituye, en definitiva, para los países ribereños a su Cuenca, un gran desafío científico que requiere de un esfuerzo conjunto e interdisciplinario. Sus componentes que la integran son Lugar de Vida - Comunidad de Vida - Política de Desarrollo Marítimo y Relaciones de Poder, cimentadas en un cuerpo compartido de Ética y Valores.

\section{El Ecocéano del Pacífico como lugar de vida}

El Ecocéano del Pacífico como Lugar de Vida se le concibe como morada y ámbito vital, donde nace y se desarrolla la actividad marítima y en cuyo espacio conviven, se interrelacionan y dependen primariamente para su desarrollo, los países ribereños que la pueblan (Geografía, aguas, subsuelos y fondos marinos).

Como ámbito vital, también lo constituye el potencial energético que lo caracteriza para su desarrollo marítimo, en cuanto a recursos marinos, vivos o no vivos, y los factores dinámicos de su entorno, naturales o políticos-culturales que lo condicionan (política de desarrollo, biomasa, plancton, minería, tecnología y factores ambientales, tales como demografía, clima, etc.).

El Ecocéano del Pacífico, percibido como Lugar de Vida en términos de morada y ámbito vital, conforman el escenario y las variables

$>$ Figura 1. Vista de la bahía de Valparaíso desde la bajada Avenida El Parque, Playa Ancha. Fotografía del autor. 
interactuantes que se manifiestan en el desarrollo marítimo de los países ribereños a su cuenca.

La noción de Ecocéano como Lugar de Vida en el Pacífico, en cuanto a su extensión, no se agota en su cuenca, sino que por imperativo ecológico se extiende a todas aquellas áreas que la afectan.

La percepción del Ecocéano del Pacífico como Lugar de Vida en función del potencial de sus recursos y el necesario equilibrio ecológico, conlleva una visión de explotación armónica y que, como un todo organizacional, en términos energéticos, busca su seguridad marítima.

El Ecocéano del Pacífico como lugar de vida asumido en términos energéticos tiene su impacto en la condición primaria de seguridad del Ecocéano y su fundamento se sustenta en la necesidad de preservar el medio marino en un mundo creciente, interdependiente y cada vez más tecnificado.

\section{El Ecocéano del Pacífico como comunidad de vida}

El Ecocéano del Pacífico en su expresión dinámica conlleva la idea de Comunidad de Vida, asumida en términos relacionales, en la que la política y el poder son sus componentes.

La expresión Comunidad de Vida, como fenómeno relacional, conlleva la idea de la necesidad de un equilibrio dinámico en los procesos de interacción que regulan la actividad marítima, y que como en el caminar encuentra su estabilidad en el movimiento, que resulta de las variables del entorno y de los efectos de sus relaciones.

En ella, las componentes interactuantes del lugar de vida surgen del escenario del Pacífico y las variables de su entorno, toman su expresión dinámica cuando los países de la cuenca generan sus actividades marítimas, en función de sus políticas de desarrollo.

En este contexto, la dinámica de la interacción marítima concebida como entramado socio-cultural de voluntades e intereses en función de sus objetivos de desarrollo, conlleva pautas de conductas que caracterizando determinadas concepciones de la realidad social, en las estrategias de sus políticas (económicas, militares, culturales, etc...), pueden tomar la forma de una relación dialéctica, consciente y activa.

Toma la forma de una relación dialéctica, cuando los objetivos de su interacción se hacen excluyentes, en cuyo caso, se generan situaciones de conflicto y de tensión social.

Toma la forma de una relación consciente, en la medida que, en las relaciones individuales y colectivas, coexistan la voluntad de acción en función de las posibilidades de desarrollo marítimo que ofrece el medio.

Toma la forma de una relación activa en la medida en que las expectativas del desarrollo de una nación marítima se vean reflejadas como objetivos en las Políticas Nacionales de Desarrollo y sean norma en el Derecho Internacional Marítimo.

Se hace evidente entonces, que, en la idea de Comunidad de Vida del Pacífico, asumida en términos relacionales, tienen significación las variables políticas, sociales, económicas ecológicas que resultan de las Políticas de Desarrollo en la interacción marítima de los Estados y que tienen su impacto en la calidad de vida marítima de los pueblos que la confluyen.

\section{La calidad de vida marítima}

Por calidad de vida marítima, entendemos al grado de satisfacción de las necesidades primarias de una nación alcanzando como re- sultado de la interacción marítima y que surgida de sus políticas de Desarrollo Nacional son asumidas en términos de una relación consciente y activa, y en un entorno de estabilidad marítima.

La Calidad de Vida Marítima de una Nación o de la Región, podemos, en consecuencia, medirla por el grado de satisfacción de las necesidades primarias de:

- SEGURIDAD REGIONAL para la estabilidad y protección del medio marino y sus recursos.

- SOLIDARIDAD REGIONAL para la articulación necesaria de una voluntad individual y colectiva en la promoción de los mecanismos de protección, explotación y usos de los recursos marítimos.

- COMPLEMENTARIEDAD REGIONAL en los objetivos de desarrollo marítimo buscados por los países de la región en la cuenca.

El Ecocéano del Pacífico, asumido en términos relacionales como Lugar de Vida, Comunidad de Vida y Calidad de Vida, tiene su impacto en las condiciones primarias de la estabilidad marítima.

\section{CONDICIONES PRIMARIAS DE LA ESTABILIDAD MARÍTIMA}

Por estabilidad marítima entendemos el clima organizacional de las instituciones, grupos o sistemas que surgiendo de las relaciones humanas en los procesos de interacción marítima y en armonía con las variables de su entorno hacen posible la satisfacción de sus necesidades de preservación, crecimiento integral y desarrollo armónico.

La estabilidad marítima, como entorno psicosocial que favorece la interacción humana en el ámbito marino es el resultado de un clima organizacional en la dinámica de sus relaciones, que conjugando los factores motivacionales que condicionan las conductas, dan base a una proyección existencial a una realidad objetiva manifestada en el desarrollo de las organizaciones marítimas y a una realidad subjetiva valorada en términos de satisfacción de las necesidades primarias individuales y colectivas.

Como condición existencial en la satisfacción de sus necesidades primarias que surgen de las funciones de preservación, integración y desarrollo, la estabilidad marítima de las organizaciones y estados participantes, presupone un entorno de seguridad, solidaridad e interdependencia funcional.

Como componentes primarios de la estabilidad marítima, las condiciones primarias de la interacción marítima, existen en una relación jerárquica y secuencial en un continum de estabilidad de la conducta, asociado a los factores motivacionales que la condicionan por vías de sus sistemas de creencias y de valores.

\section{EL CONCEPTO DE SEGURIDAD ECOLÓGICA COMO CONDICION PRIMARIA DE LA ESTABILIDAD MARÏTIMA}

La seguridad como componente básico de las condiciones primarias de la estabilidad social, se sitúa en el nivel de preservación del cuerpo social, y como tal su materialización responde a factores motivacionales de naturaleza biopsíquica consustancial a las necesidades energéticas de los sistemas vivos.

En su expresión energética, la seguridad marítima entendida como ausencia de riesgo, se constituye en el componente básico de la estabilidad social y precondición de la solidaridad humana que basada en los deberes y el respeto a las normas jurídicas posibilita la interacción marítima en un clima de confianza mutua requerido por 
los individuos, grupos y sistemas en su función de integración, y en ella se fundamenta la interdependencia funcional requerida por el cuerpo social para su función de desarrollo.

La seguridad ecológica asumida en términos dinámicos como resultado de la interacción marítima es consecuencia de un modelo ético sustentado en principios valóricos que promueven un enfoque integral del hombre en armonía con su ambiente. Como entorno de estabilidad que surge de la responsabilidad individual y conciencia colectiva, la seguridad ecológica se fundamenta en un diario vivir anclado en los deberes morales que promueven una convivencia existencial valorada en términos de calidad de vida.

La seguridad ecológica como entorno de estabilidad existencial se materializa en todos los ámbitos del quehacer humano, y en su dimensión psicosocial se la percibe en términos de efectos en función de sus inseguridades.

En su deterioro, como proceso de desestabilización existencial que afecta a un todo relacional y de responsabilidad compartida, son determinantes las conductas irresponsables, o de otro modo delictuales, que socavando las bases de sustentación energética que posibilitan la vida en el planeta, comprometen gravemente los derechos de las generaciones presentes y futuras.

La protección del medio ambiente acuático, en ella, el de la Cuenca del Pacífico, y la Zona Antártica y Preantártica, tiene una relación directa e inmediata con el rol de la Defensa y una Política Nacional que la concrete.

El rol de la Defensa, en la perspectiva dinámica del Poder Político, comienza mucho antes que la agresión y en ella concurren todos los ámbitos del quehacer nacional y su materialización presupone ámbitos de competencia y roles de acción en el plano de la vigilancia, control, prevención, disuasión y reacción, por lo que su dimensión excede los límites de lo estrictamente militar.

En su función de Preservación está el de anticiparse a la ocurrencia de los hechos en resguardo de las variables que la amenacen o puedan afectarla. De aquí que en lo referido a la protección ecológica y ambiental y el Rol Presencial de la Defensa en su acción de precaver recae esencialmente en la anticipación a la posible ocurrencia de hechos que pudieran afectar al medio ambiente.

La acción de anticipación constituye en consecuencia la principal forma que tiene el Rol de la Defensa para preservar el patrimonio ecológico y el medio ambiente considerando que los efectos de todo potencial daño que pudiera ocurrir no solo importa un compromiso en su dimensión espacial dada por el área afectada sino también en su dimensión temporal dice relación con el tiempo requerido para reparar daños ecológicos lentos en sus procesos y muchas veces irreversibles.

\section{FILOSOFÍA POLITICA: EL LEGADO CULTURAL HISPÁNICO}

\section{Percepción Vitoriana en las Relaciones de Poder}

La filosofía política que en la dinámica de cambios otorga a la realidad emergente un marco de convivencia pacífica como orden jurídico internacional, fue formulada en el siglo XV por Francisco de Vitoria cuyos principios puso en marcha un nuevo estilo de vida y de convivencia pacífica que enraizada en el derecho natural y el derecho de gentes ofrecen a la comunidad internacional un anclaje moral que iluminan las nuevas formas de convivencia e integración.
Basado en la universalización de la paz como un todo indivisible y solo condicionada por la justicia y la libertad, los principios vitorianos que encauzan la conducta humana se fundan en el respeto mutuo y la solidaridad sustentada en la tesis de la paz dinámica, entendida como paz armada en un contexto relacional de políticas e intereses en defensa de los valores nacionales, sus soberanías y los principios universales de la humanidad.

En este contexto la paz armada se convierte para Vitoria tanto como la moral y el derecho, en condición indispensable de un sistema de intereses y equilibrio en la que la política y el poder son los componentes dinámicos de su interacción.

Poder, que expresado en término de capacidades, atributos y alcance legitimado en el derecho internacional se transforma en herramienta de integración y desarrollo.

Política, que, imbuida en valores de justicia, respeto y equidad sustentada en una moral universal, promueven un clima de estabilidad social en beneficio de la paz y la cooperación internacional.

La paz armada, así concebida por Vitoria como parte integrante del poder político, en su tarea permanente de asegurarla en desarrollo, se constituye en la componente militar del poder del estado, como instrumento bélico para el resguardo de las condiciones de estabilidad que surgen del estado de derecho otorgándole su eficacia respaldado en su capacidad de fuerza y de acción.

En el legado de Vitoria y sustentado en los principios de paz dinámica, como lo ha denominado el catedrático español Luciano Pereña ${ }^{2}$, en el corpus hispanorum de pace, encontramos la filosofía política que otorga a la sociedad universal el marco de convivencia pacífica basada en la universalización de la paz, condicionada por la justicia y la libertad, y el fundamento doctrinario para interpretar el rol de las fuerzas armadas en su tarea permanente de asegurar la paz.

La política y el poder, así concebida en términos relacionales en un contexto de paz dinámica y cimentada en los principios vitorianos, que promueven una moral universal, presuponen en consecuencia, para su desarrollo orgánico un estado de equilibrio dinámico que exige de los gobernantes y las instituciones una revisión y readecuación permanente de sus estructuras y mecanismos de protección.

\section{PERSPECTIVA DINÁMICA DEL PODER}

Planteado el fenómeno de la globalización y los efectos asociados a una realidad emergente, que en el horizonte del tercer milenio, confronta la realidad existencial con las variables de su entorno y el desafío de un desarrollo integral en un contexto de estabilidad social, se hace necesario, visualizar la interacción de los Estados, desde una perspectiva vitoriana, en función de objetivos complementarios de desarrollo en el contexto de las relaciones de poder y que en el ámbito internacional tienen un carácter político-estratégico, socio-cultural y económico, y que plantean la necesidad de una redefinición.

Una primera aproximación, requiere observar el poder no como algo estático que se pueda tener, sino como una condición dinámica y relacional que se debe reconocer como la causa de efectos deseados resultantes en la interacción en un contexto de estabilidad social y de desarrollo armónico e integral.

Poder no es tener, sino hacer. El Poder se ejerce en el marco de las relaciones humanas y se expresa en término de capacidades y efectos a través de las funciones de Preservación, Integración y Desarrollo que ocurren en la interacción social. 


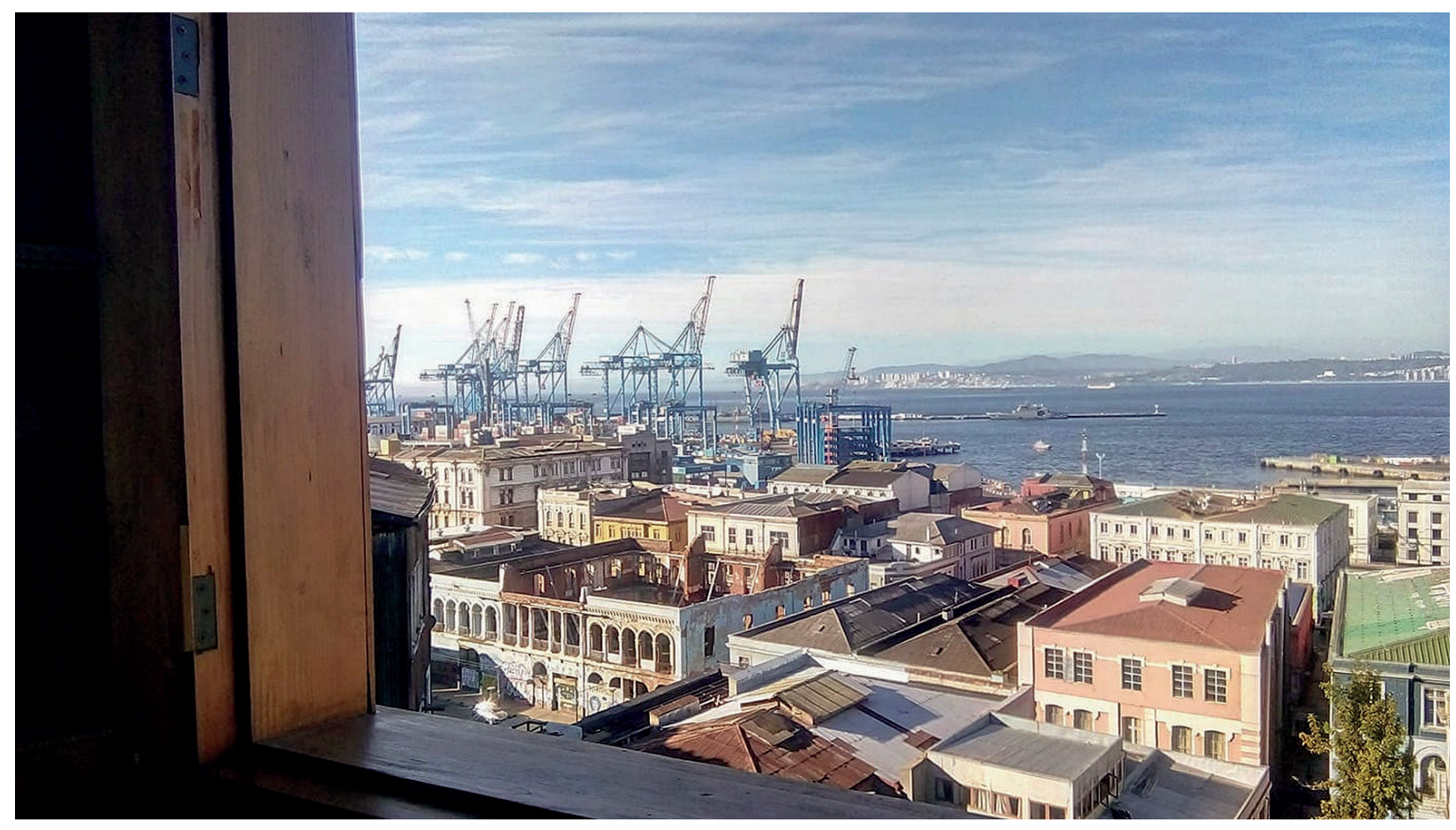

Como una forma relacional, el Poder tiene su impacto en la calidad de vida de las naciones, manifestadas en las condiciones de seguridad, solidaridad, y complementariedad de los estados y que son primarias en los sistemas sociales.

El Poder como fuente de acción no existe en el vacío y en su manifestación política como última instancia a nivel societal se ve asociada a la soberanía de los estados.

Como expresión relacional, el Poder político presupone un escenario político cultural y un contexto social en el que se desarrolla.

Desde la perspectiva Vitoriana y asumiendo el concepto del Poder Político como la capacidad que tienen los estados para alcanzar las metas de su desarrollo en un contexto de justicia, integración y paz universal cabe preguntarse, lo ya afirmado por Jean Ladriere $^{3}$ en su ensayo sobre el poder, si acaso una teoría completa del poder en su expresión política como fuente absoluta de acción de un estado, no requiere de una norma de acción de naturaleza moral exterior a él.

\section{Estructuración Vitoriana del poder}

Desde esta perspectiva visualizamos el Poder como componente dinámico de la interacción humana en función de objetivos complementarios nacionales y en armonía con el desarrollo regional y universal en la comunidad de las naciones, expresados en las dimensiones sociales de energía, estructura y función.

El poder operacional, según situemos el poder en el ámbito de las potencialidades, en cuanto a conocimientos, medios y recursos, y que, expresados en campos de acción, se agrupan genéricamente en lo económico, psicosocial, diplomático y militar. En su dimensión energética, el poder operacional dice relación con la capacidad de los estados para alcanzar las metas de su desarrollo en armonía con los objetivos regionales y universales y en equilibrio con las variables naturales de su entorno.

El poder transaccional, según se perciba el poder en el ámbito de sus relaciones en cuanto a sus atributos; de naturaleza activa, disuasiva, o defensiva expresada en sus políticas de desarrollo económico, social, exterior y de defensa. El poder transaccional,

$>$ Figura 2. Vista del puerto y ciudad de Valparaíso desde el mirador subida Serrano. Fotografía del autor. 
en su dimensión estructural, dice relación con los mecanismos de integración que en función de objetivos nacionales complementan su acción para alcanzar las metas de su desarrollo.

El poder inductivo, según se perciba al poder en el ámbito de sus alcances, dada por la naturaleza de su transacción consensual o coercitiva y en los efectos de su normativa; de mantención, de modificación o de cambio en función de su desarrollo. El poder inductivo, en su dimensión funcional, dice relación con los objetivos nacionales, regionales y universales que materializados en las condiciones primarias de seguridad, solidaridad y complementariedad dan cabida existencial al mundo natural y al social, como parte de un todo de responsabilidad humana y compartida.

\section{DESARROLLO OCEÁNICO INTEGRAL}

Asumida la Cuenca del Pacífico como un todo existencial que integra la idea de Lugar de Vida, Comunidad de Vida y Calidad de Vida, y estructurada las componentes del poder de la actividad marítima en términos relacionales desde una visión oceanopolítica en función de su preservación, integración y desarrollo, identificamos el cuerpo de conocimiento que, en sus respectivos ámbitos del saber, conforman el concepto de desarrollo oceánico integral:

- EN SU DIMENSIÓN ENERGÉTICA y en función de sus recursos, explotación y preservación, en equilibrio con su entorno, identificamos un cuerpo de conocimientos, que por aportar a la función de preservación, en términos de calidad de vida marítima definimos como ecocéano. El Ecocéano en cuanto conocimiento centrado en la potencialidad de los recursos marítimos de una región y expresado en la forma de poder operacional de los estados en equilibrio con su entorno se manifiesta en el grado de seguridad marítima.

- EN SU DIMENSIÓN ESTRUCTURAL y en función de la actividad marítima a través de su administración, financiación y producción, identificamos un cuerpo de conocimientos que definimos como oceanotecnia. La Oceanotecnia en cuanto vías de acción centrada en la interacción marítima de los Estados a través de los servicios, usos y explotación de los recursos marítimos de una región se expresa en la forma de poder transaccional por el grado de actividad marítima que realiza un Estado.

- EN SU DIMENSIÓN FUNCIONAL y concurrente con los objetivos nacionales de una política oceánica de desarrollo, asumida en términos relacionales y desde una perspectiva integral, a través de políticas marítimas, su estrategia y los planes de acción que la materializan, identificamos el cuerpo de conocimientos que se entiende como oceanopolítica. La Oceanopolítica, como aproximación integral que incorpora el mar en la estructura del espacio político desde una perspectiva relacional en función de objetivos de desarrollo nacional y regional, se expresa en la forma de poder inductivo por el grado de desarrollo integral y estabilidad lograda en el usufructo de los intereses marítimos.

\section{FUNDAMENTO ÉTICO DEL DESARROLLO MARÍTIMO}

El pensamiento de Vitoria que surge como juicio crítico del proceso a la conquista de América, centrado en los derechos del hombre y la dignidad del indio, por la profundidad de su mensaje enraizado en la conciencia y solidaridad de los pueblos toma forma orgánica y su desarrollo, como conciencia de américa y ahora universal, se manifiesta latente en los momentos decisivos de la historia americana.
Los principios Vitorianos fueron recogidos por los acuerdos americanos, dando cimiento al principio de igualdad jurídica de los Estados, el de no intervención y el de no reconocimiento de la adquisición por la fuerza, con que, incorporados en Chapultepec, fueron tomados de la primera conferencia internacional americana.

Como elemento de continuidad histórica en el ámbito de lo marítimo, el desarrollo de la conciencia americana, estructurada en los principios de Vitoria, no está ausente del esfuerzo regional de los países hispanoamericanos. En particular, Chile, ha contribuido a materializar en el derecho internacional marítimo, los principios morales que regulan la convivencia de la comunidad de las naciones, en resguardo de los intereses marítimos regionales y nacionales.

Señaladas como la respuesta de Chile al desafío oceánico por el Sr. Almirante Jorge Martínez, en su clase magistral La gran tarea de esta generación es la ocupación efectiva de nuestro mar, identificamos los siguientes principios jurídicos inspirados en los principios Vitorianos y que hoy son normas en el Derecho Internacional:

a. La creación del concepto de Zona Contigua incorporado por Chile en el Código Civil Chileno de 1855, y que dio origen al concepto jurídico de Mar Territorial.

b. La Declaración Oficial del Presidente del Gobierno chileno sobre jurisdicción marítima, que en 1947 declaró la soberanía nacional sobre el zócalo continental e insular y sobre los mares adyacentes a las costas de Chile, hasta una distancia de 200 millas y que dio origen al concepto jurídico de Plataforma Continental, incorporado en el derecho del mar de las Naciones Unidas.

c. La Declaración de Santiago de Chile, de los gobiernos de Chile, Ecuador y Perú que, en 1952, proclamaron como norma de su política internacional marítima, la soberanía y jurisdicción exclusiva sobre los espacios marítimos hasta las 200 millas y que da origen al concepto jurídico de la Zona Económica Exclusiva.

Asimismo, la proposición del concepto de Mar Presencial elaborado por el Sr. Almirante Jorge Martínez, que define espacios marítimos en términos de los intereses nacionales, sin alterar la soberanía marítima de los Estados y que ofrece a la comunidad de las Naciones un mecanismo de integración regional en resguardo de sus intereses marítimos, constituye un aporte cultural marítimo en la línea del pensamiento ético, cuya iniciativa ha tomado eco en el ámbito legislativo a través de la normativa que regula la ley de pesca y en el ámbito académico como primer acuerdo de un congreso nacional a iniciativa de la comunidad académica de la Ingeniería Naval Marítima

En efecto, las conclusiones que el Capítulo Chileno del Instituto Panamericano de Ingeniería Naval entregó a la Comunidad Académica Nacional e Internacional como resultado de su Primer Congreso Nacional de Ingeniería Naval y Marítima en su Proyección Oceánica, así lo demuestran cuando en sus Considerandos estipula:

- Que la idea y el concepto del mar presencial debiera ser la inspiración que oriente el quehacer de sus miembros en sus actividades hacia el desarrollo naval y marítimo.

- Que el concepto de mar presencial se encuentra inserto en una visión sistémica oceánica integral, en la cual la Ingeniería Naval forma parte en el ámbito de la tecnología, constituyendo un elemento de continuidad histórica en el desarrollo nacional y que dispone de la capacidad potencial para hacer posible esta realidad. 
- Que se propone contribuir a que la iniciativa por el mar presencial sea acogida por la Ingeniería Naval Panamericana, y en especial por la de los países de la comunidad del Pacífico Sur en función de los intereses marítimos comunes y nacionales en la Región.

\section{REFLEXIONES FINALES}

En el horizonte del siglo XXI, hoy, más que nunca se plantea a la comunidad universal, el desafío de asumir la dinámica de cambios que caracteriza a una realidad social acelerada, desde una perspectiva de desarrollo integral y armónico y en un clima de justicia y de paz social.

El fenómeno de la globalización y su realidad emergente asociado a una toma de conciencia en las condiciones existenciales que hoy afectan a la humanidad, plantean el desafío de asumir la realidad existencial desde una perspectiva sistémica e integral y sustentada en principios valóricos universales que la contengan.

La comunidad del Pacífico, percibida como un todo relacional, no está ajeno a ello; su desarrollo marítimo, desde una perspectiva oceánica integral, en un contexto de paz dinámica, en el que se mantenga la diversidad de sus componentes, configura hoy la exigencia del presente, para edificar los cauces del futuro.

La Visión Sistémica de lo marítimo y los fundamentos éticos que la contienen sustentadas en los principios de Vitoria, ofrecen a la comunidad naval y marítima una vía integral de desarrollo.

\section{NOTAS}

1. M. Marinovic, Análisis para un Desarrollo Marítimo-Oceánico Integrado, desde la Perspectiva Vitoriana o Escuela Española de la Paz. Ponencia presentada en el Congreso Internacional de Universidades 1992, "La Universidad ante el Quinto Centenario", Madrid, julio de 1992.

2. Luciano Pereña, Francisco de Vitoria, Colección Corpus Hispanorum de Pace.

3. Jean Landriere, Poder y Conflicto, Editorial del Pacífico, Instituto de Estudios Políticos, 1975. 